\title{
Foreword
}

\section{Global Strategies for Disease Detection and Treatment: Proteomics}

\author{
Sudhir Srivastava ${ }^{\mathrm{a}}$ and Sam Hanash ${ }^{\mathrm{b}}$ \\ ${ }^{a}$ Cancer Biomarkers Research Group, Division of \\ Cancer Prevention, National Cancer Institute, \\ Bethesda, MD 20892, USA \\ ${ }^{\mathrm{b}}$ University of Michigan Medical Center, Ann Arbor, \\ MI 48109, USA
}

The field of proteomics holds great promise for identifying nontargeted, global molecular profiles "signatures" of normal and diseased cells. While genomics offers a wide array of tools for identifying mutated or dysregulated genes, proteomics offers the ability to measure post-translational modifications, protein stability, phosphorylation state, protein-protein interactions, and protein DNA-binding affinities. These biological events play major roles in the pathogenesis of disease and cannot be studied by DNA and mRNA efforts alone. Recent studies have shown that there is no specific correlation between mRNA abundance and protein expression levels in a cell at a given time. The discrepancy could arise from the control of mRNA translation, and the stability of mRNAs and proteins. Because almost all therapeutic intervention strategies or early detection technologies target expressed proteins, proteomic-based studies can provide fundamental information to characterize disease progression at the molecular level. Therefore, proteomics has particular relevance to diagnostics and to the identification of disease markers, as proteins can be assayed in serum and other biological fluids.

Since the completion of human genome sequence, the discipline of proteomics has taken a center stage in defining the future of molecular diagnostics. This is due, to some extent, to the fact that the sequencing of the human genome and other important genomes has opened the door for proteomics by providing a sequence-based framework for mining the proteome. The growth in proteomic tools and proteomics is in an extraordinary growth phase. As a consequence, pro- teomics is witnessing an extraordinary growth in the number of new investigators and biotechnology companies that are taking an active interest in the field. Proteomics is evolving at a fast pace, as is evident from this special issue. Strategies for protein fractionation prior to analysis are increasingly being relied upon. Selective enrichment for a subset of proteins of interest can be achieved using a variety of techniques from centrifugation procedures to affinity capture. In addition to reducing sample complexity and increasing sensitivity, strategies based on the separate analysis of subcellular compartments provide the means to determine protein location in a cell. There is clearly interest in developing robust "industrial strength" proteomic platforms to achieve high-throughput and high sensitivity.

During the lean years of proteomics, the field was largely dominated by a single approach, namely twodimensional (2-D) gels, backed up by an assortment of related tools, ranging from software for image analysis to mass spectrometry. Several technologies for protein profiling are emerging that are not 2-D gel based, including direct profiling by mass spectrometry and the use of protein microarrays. High throughput technologies, such as Surface-Enhanced Laser Desorption/Ionization Time-of-Flight (SELDI-TOF) and Matrix-Assisted Laser Desorption/Ionization Time-ofFlight (MALDI-TOF) technologies, are providing us with a non-biased, global discovery approach using patient serum, plasma, urine or other sources of secretions, to identify - in a single run - the expression patterns in thousands of small molecular mass proteins $(<20 \mathrm{kDa})$ based on their molecular mass and charge, offering a tool to determine which proteins are secreted from tumor cells or are measurable in the bodily fluids of patients. However, it is likely that no one technology platform will meet the needs of the wide spectrum of applications encompassed by proteomics. Diagnosing disease through proteomics may well require all the tools at our disposal to identify and validate the most promising markers. 
While the emergence of proteomics brings hope to disease detection and therapeutic intervention, it poses several analytical challenges. Analyzing voluminous data generated by high-throughput proteomics arrays is a fairly new endeavor for statisticians for which there is not extensive literature. Another complexity to these analyses is how to analyze proteomics data in reference to disease outcomes. The maturation of proteomics technology, with its global, non-directed ability to analyze serum proteins, will add to the markers or patterns of markers that are able to predict the presence of ovarian cancer. For example, if the protein products of the amplified DNA or RNA are secreted from tumor cells and migrate to serum, plasma, urine, or other accessible fluids, proteomics will offer a rapid advance in the identification of novel biomarkers to facilitate the development of noninvasive, sensitive assays for earlier cancer detection.

This special issue of Disease Markers covers all aspects of proteomics with special emphasis on clinical applications. Some of the proposed approaches may not be ready for a clinical use, however, they are justified and desirable in the spirit of the developing field of proteome research. 


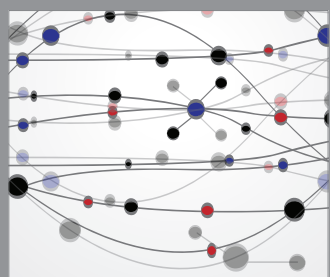

The Scientific World Journal
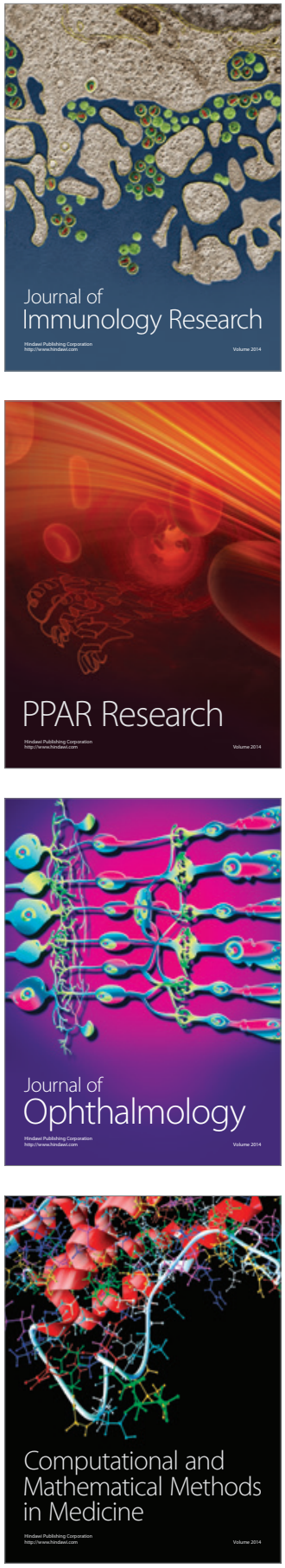

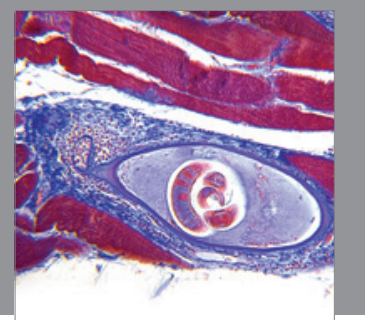

Gastroenterology

Research and Practice
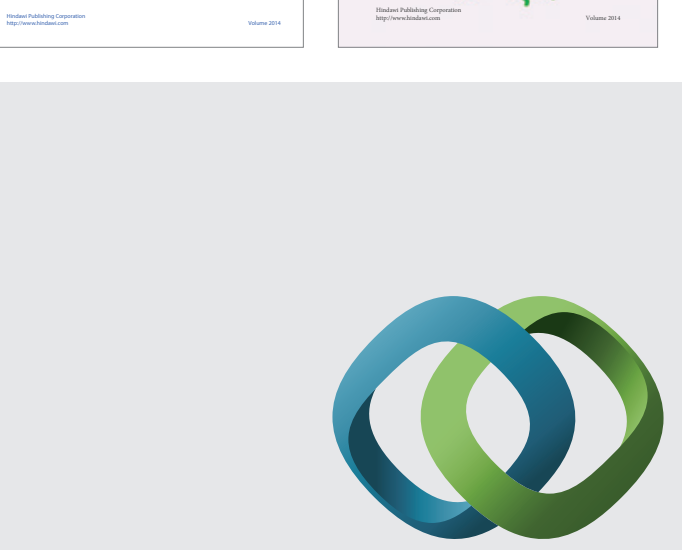

\section{Hindawi}

Submit your manuscripts at

http://www.hindawi.com
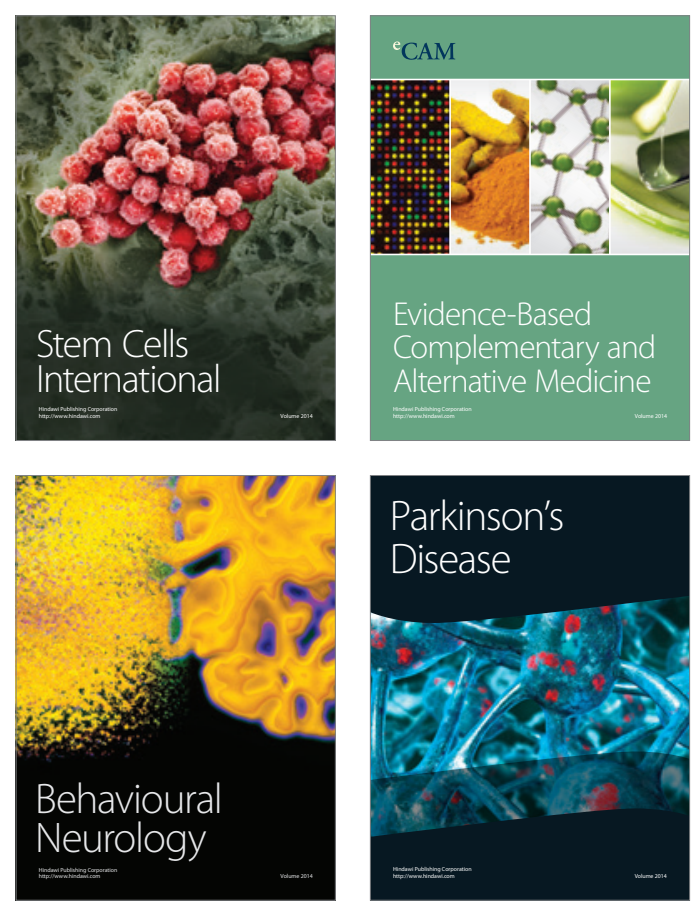

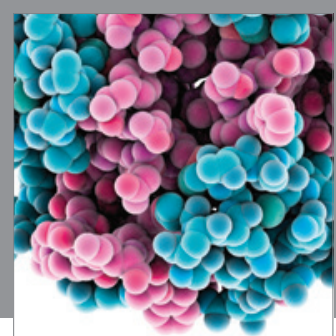

Journal of
Diabetes Research

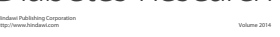

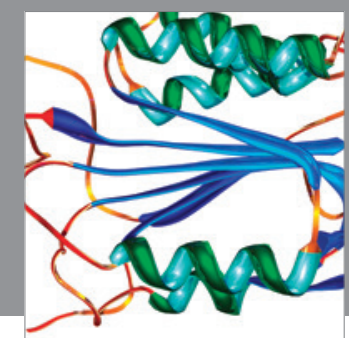

Disease Markers
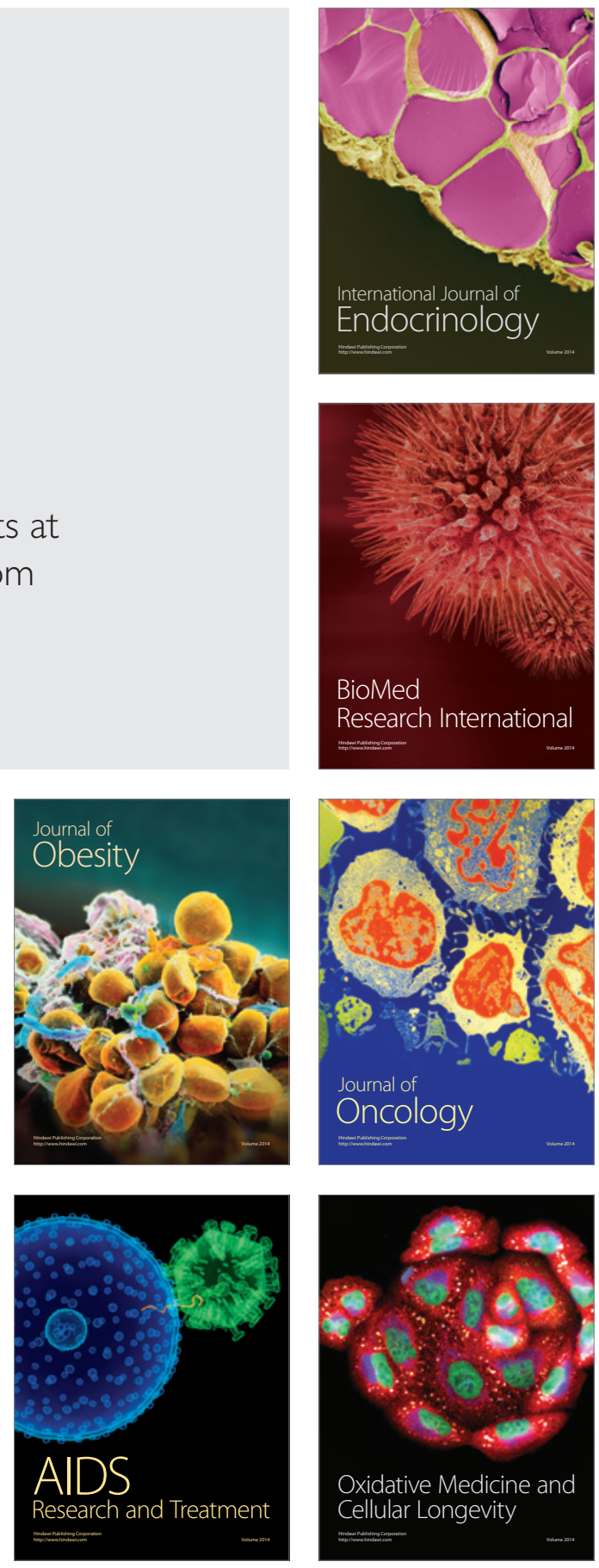